\title{
A Model for Glioma Growth
}

\author{
EVGENIY KHAIN, ${ }^{1}$ LEONARD M. SANDER, ${ }^{1}$ AND ANDREW M. STEIN ${ }^{2}$ \\ ${ }^{1}$ Department of Physics and Michigan Center for Theoretical Physics, The University of Michigan, Ann Arbor, \\ Michigan 48109; and ${ }^{2}$ Department of Mathematics, The University of Michigan, Ann Arbor, Michigan 48109
}

This paper was submitted as an invited paper resulting from "Understanding Complex Systems" conference held at University of Illinois-Urbana Champaign, May 2005

Received September 30, 2005; accepted November 16, 2005

\begin{abstract}
Glioblastoma Multiforme (GBM) is the most invasive form of primary brain tumor. We propose a mathematical model that describes such tumor growth and allows us to describe two different mechanisms of cell invasion: diffusion (random motion) and chemotaxis (directed motion along the gradient of the chemoattractant concentration). The results are in a quantitative agreement with recent in vitro experiments. It was observed in experiments that the outer invasive zone grows faster than the inner proliferative region. We argue that this feature indicates transient behavior, and that the growth velocities tend to the same constant value for larger times. A longer-time experiment is needed to verify this hypothesis and to choose between the two basic mechanisms for tumor growth. (C) 2005 Wiley Periodicals, Inc. Complexity 11: 53-57, 2005
\end{abstract}

Key Words: tumor growth; invasion; proliferation; diffusion; chemotaxis; mathematical modeling

\section{INTRODUCTION}

T his article reports on an analysis of recent experiments (Stein et al., unpublished results) on the in vitro growth of Glioblastoma Multiforme (GBM). GBM is a highly malignant brain tumor, the most common and the most aggressive of the primary brain tumors. Despite major improvements in cancer treatment, the overall prognosis is still very poor $[1,2]$. Such a high mortality and poor response to treatment is due the fact that GBM is highly invasive [3].

Correspondence to: Leonard M. Sander, Randall Laboratory of Physics, University of Michigan, Ann Arbor, MI 481091120.E-mail: lsander@umich.edu
Tumor cells are able not only to proliferate but also to migrate in the extracellular matrix of the brain. (Note that migration is not the same as metastasis, where cancer spreads in the circulatory system.) A typical tumor consists of an inner proliferation zone with a very high density of cells and a larger outer invasive zone, where the cell density is smaller (see Figure 1). Surgery can remove only the inner solid tumor, but not the cells in the invasive zone, so that secondary (recurrent) tumors are very common [3].

The growth of brain tumors in vivo is a complex biological phenomenon. Therefore, many efforts were made to investigate model systems where tumors grow in vitro. In recent experiments, in vitro tumor growth starting with tumor spheroids was investigated in transparent gels [4]. 


\section{FIGURE 1}

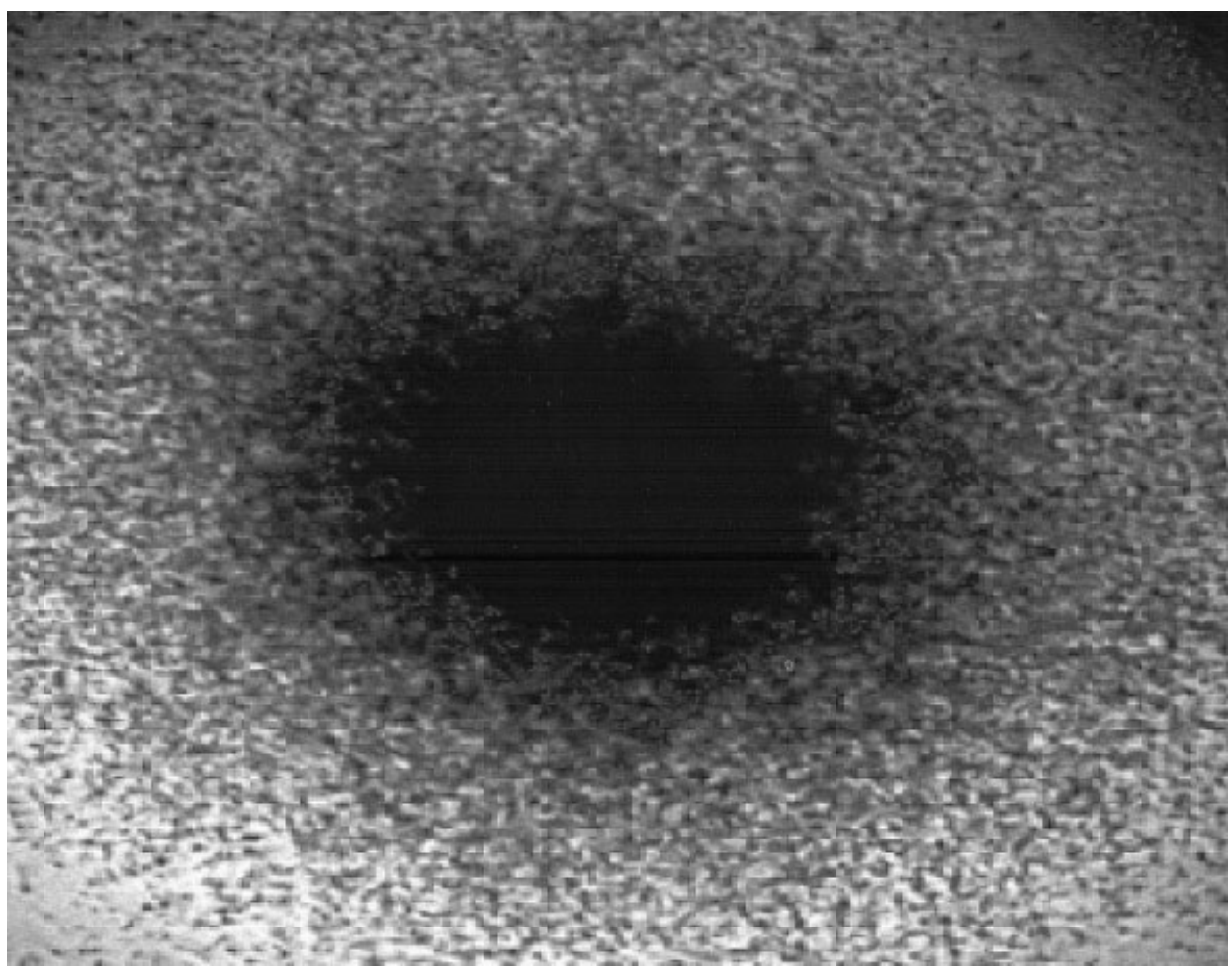

Example of growing tumor from in vitro experiments in collagen gel (Stein et al., unpublished results). There is an inner proliferation zone with a very high density of cells and a larger outer invasive zone, where the cell density is smaller. The diameter of the inner core is about $250 \mu \mathrm{m}$.

The experimentally observed branching patterns can be explained theoretically by homotype attraction [5]. However, these branching patterns are not always observed experimentally. For one of the cell lines studied (U87 wild type) experiments showed a spherically symmetric growing tumor (Stein et al., unpublished results). This is the case we will consider here.

Though the cells within the invasive and proliferative region have the same genotype, their phenotype is quite different. The invasive cells migrate faster and have a lower proliferation rate (Stein et al., unpublished results) than those in the tumor core. This dichotomy between migration and proliferation regimes was first observed by Giese and coauthors [6,7]. To model these experimental observations, Stein and coauthors (Stein et al., unpublished results) proposed a two population model. In this scheme there are densely packed cells within the tumor core and invasive cells that are continuously shed from the surface of the tumor. The approach we take in this work is different. Here, we formulate a simple physical model with only one type of cells and describe the dynamical switching of the cell phenotype by a density-dependent diffusion coefficient. We include in two different possible mechanisms of tumor in- vasion: diffusion (random motility) and chemotaxis (directed motility).

In the next section we describe the model. Then we present the results and consider two basic mechanisms that lead to tumor invasion: diffusion and chemotaxis. The last section includes a brief discussion and a summary.

\section{MODEL AND GOVERNING EQUATIONS}

The basic unit in our description will be a cancer cell. Consider an initial tumor in a three-dimensional medium. Each cell is able to proliferate and to perform random motion (diffusion) and guided, directed motion (chemotaxis). Chemotaxis is the directed motion of a cell along the gradient of a chemoattractant concentration. The chemoattractant could be one of several chemical agents such as glucose or various growth factors. In our model, these molecules bind to cell receptors. The gradient of chemoattractant concentration is caused by consumption by the growing tumor. As the result, a cell moves away from the tumor.

The governing equations for the cell concentration $u(\tilde{r}, t)$ and for the concentration of chemoattractant $c(\tilde{r}, t)$ are as follows: 


\section{FIGURE 2}

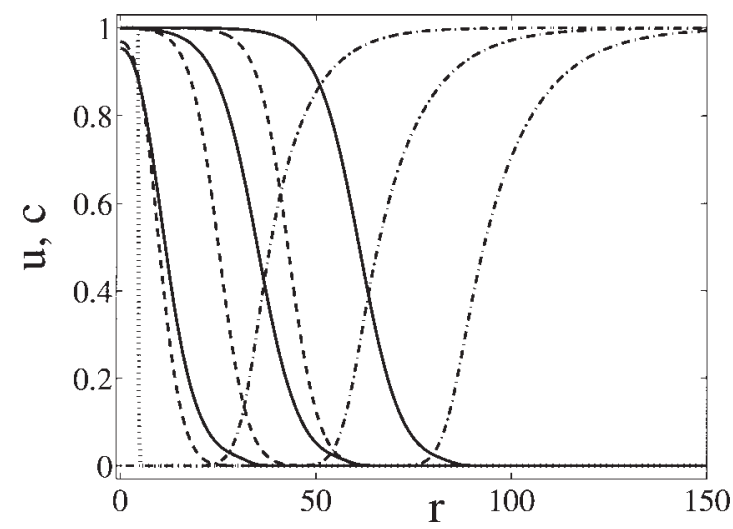

Time series of the density profiles of cells and of chemoattractant, obtained from the numerical solution of Eq. (2). We start with a sharp cell density profile (dotted line). After a short transient, one can see two propagating fronts: the first one represents the growing tumor (cell concentration, solid lines), the second one represents the consumed chemoattractant (chemoattractant concentration, dash-dotted lines). The profiles correspond to times $t=5, t=10$, and $t=15$. The parameters are $D=100, \chi_{1}=10, u_{1}=0.25, c_{1}=0.3$, $\beta=700$, and $r_{0}=4.5644$. Also shown is an example of a pure diffusion front (cell concentration, dashed lines). It is obtained by putting $\chi_{1}=0$ in the first of Eqs. (2). For the same diffusion coefficients, the traveling front with diffusion and chemotaxis terms moves faster.

$$
\begin{aligned}
& \frac{\partial u}{\partial t}=\nabla \cdot\left(D_{1}(u) \nabla u\right)+g u\left(u_{c}-u\right)-\nabla \cdot(\chi(c) u \nabla c), \\
& \frac{\partial c}{\partial t}=\nabla \cdot\left(D_{2} \nabla c\right)-\alpha u c .
\end{aligned}
$$

Here $D_{1}$ and $D_{2}$ are the diffusion coefficients of cells and of chemoattractant, $\chi$ the chemotaxis coefficient, $u_{c}$ the density of the close packed cells, $g$ the rate of proliferation, and $\alpha$ the rate of consumption of $c$. We assume that the cell diffusion coefficient $D_{1}$ depends on cell concentration. This assumption is based on experimental observations that showed that cell motility is larger in the invasive region, where cell concentration is smaller [6,7]. We put $D_{1}=$ $D_{0}\left(u+u_{0}\right)^{-1}$, so that $D_{0} / u_{0}$ is the diffusion coefficient far from the tumor. The chemotaxis coefficient is given by the receptor law: $\chi=\chi_{0}\left(c+c_{0}\right)^{-2}[8]$. Chemotaxis decreases as the chemoattractant concentration increases since for large $c(\tilde{r}, t)$ most of the cell receptors are saturated, and the cell is not able to detect the chemical gradient. We assume an infinite reservoir of chemoattractant, so that $c(\tilde{r}, t)$ is constant far from the tumor $c=c_{\infty}$.

We introduce dimensionless variables. Measuring cell density in the units of $u_{c}$, chemoattractant concentration in units of $c_{\infty}$, time in units of $\left(g u_{c}\right)^{-1}$, and distance in units of $D_{0} /\left(g u_{c}\right)^{2}$, we arrive at

\section{FIGURE 3}

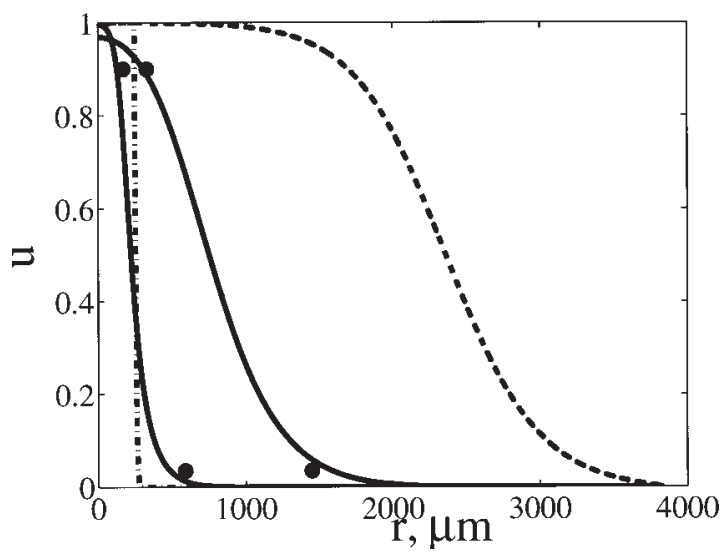

Time series of the cell density profiles in the case of pure diffusion. An initially sharp density profile is shown by the dash-dotted line. Due to diffusion effects, the front becomes smoother with time; the profiles after 1 day of tumor growth $(t=0.86)$ and 7 days $(t=6)$ are shown by the solid lines. One can see that the small density invasive region expands faster than the large density proliferative zone, in agreement with experimental data (Stein et al., unpublished results; the circles). However, this occurs only in a transient regime. Also shown is the density profile after 14 days $(t=12)$ (the dashed line). As time increases, the expansion velocities of the two zones tend to the same value. For convenience, the distance from the center of tumor is measured in physical units $(\mu \mathrm{m})$. The parameters are: $\chi_{1}=0, u_{1}=0.1$.

$$
\begin{aligned}
& \frac{\partial u}{\partial t}=u(1-u)+\nabla \cdot\left(\frac{\nabla u}{u+u_{1}}\right)-\chi_{1} \nabla \cdot\left(\frac{u \nabla c}{\left(c+c_{1}\right)^{2}}\right) \\
& \frac{\partial c}{\partial t}=D \nabla \cdot(\nabla c)-\beta u c .
\end{aligned}
$$

Here, the parameter $\chi_{1}=\chi_{0} /\left(D_{0} c_{\infty}\right)$ measures the relative strength of chemotaxis and diffusion, $D=D_{2} u_{c} / D_{0}$ is the scaled ratio of cell and chemoattractant diffusion coefficients, $\beta=\alpha / g$ is the ratio of proliferation and consumption rates, and $u_{1}=u_{0} / u_{c}, c_{1}=c_{0} / c_{\infty}$ are two parameters that lie in $[0,1]$.

\section{DIFFUSION VERSUS CHEMOTAXIS}

We assume radial symmetry and analyze a typical solution of Eqs. (2): $u=u(r, t), c=c(r, t)$. First, we need to specify boundary conditions. At $r=0$ we have

$$
\frac{\partial u}{\partial t}=\frac{\partial c}{\partial t}=0
$$

Far from the tumor, the cell concentration is zero, $u(r=$ $\infty)=0$, and the scaled chemoattractant concentration is unity, $c(r=\infty)=1$. We start with uniform chemoattractant concentration $c(r, t=0)=1$ and with an initially spherical 
solid tumor. We assume also that the cells within this tumor are densely packed: $u\left(r<r_{0}, t=0\right)=1, u\left(r>r_{0}, t=0\right)=0$. In Figure 2 we give a time series of the density profiles of cells and of chemoattractant from Eq. (2). After a short transient, there are two propagating fronts. The first front is the growing tumor: the cell density $u(r, t)$ is shown by the solid lines. The second front represents the consumed chemoattractant: the chemoattractant concentration $c(r, t)$ is shown by the dash-dotted lines. Figure 2 also gives an example of a pure diffusion front (cell concentration, dashed lines) obtained by putting $\chi_{1}=0$ in the first of Eq. (2). For the same diffusion coefficients, the traveling front of the full problem [Eq. (2)] with diffusion and chemotaxis moves faster, as expected.

The propagating front solution means that the inner proliferation region grows with the same velocity as the outer invasive zone. However, in experiments, the invasive zone grows faster than the proliferation region (Stein et al., unpublished results, [4]). This suggests that the experiments (Stein et al., unpublished results) must be in a transient regime. Indeed, any initially sharp front becomes smoother with time due to diffusion effects. It means that the region with small density moves faster than the large density zone, as is seen in Figure 3 for sufficiently small times. Another experimental confirmation (Stein et al., unpublished results) of this diffusion-based argument is the fact that at small times the tumor core shrinks, in agreement with Figure 3. To verify these predictions, a longer-time (2-3 weeks) experiment would be needed.

We can calculate the experimental radial density profile from the image shown on Figure 1. First we compute the brightness of the image $\bar{u}$ as a function of the distance from the tumor center $\rho$ in cylindrical coordinates. Then we assume that the original light intensity decays exponentially:

$$
\bar{u}(\rho)=I_{0} \int e^{-u(r)} d z
$$

Then, inverse radon transformation procedure (performed in Matlab) allows us to obtain the experimental density profile $u(r)$. Figure 4 shows both the experimental density profile from Figure 1 and two theoretical profiles, computed from the numerical solution of Eq. (2), which correspond to the two different scenarios. The first one includes both diffusion and chemotaxis, whereas the second one includes only diffusion (the diffusion coefficients are different in the two cases.) Despite the similarity between the two theoretical density profiles in the early stage (both of them are in a good agreement with the experimental profile), the two scenarios are distinguishable at larger times. It should be noted that when grown in spinner flasks, tumor spheroids stop growing when they reach $1-2 \mathrm{~mm}$ in radius [9]. This phenomenon is likely due to mitotic inhibitors that are produced by the necrotic core of the spheroid [9]. Our

\section{FIGURE 4}

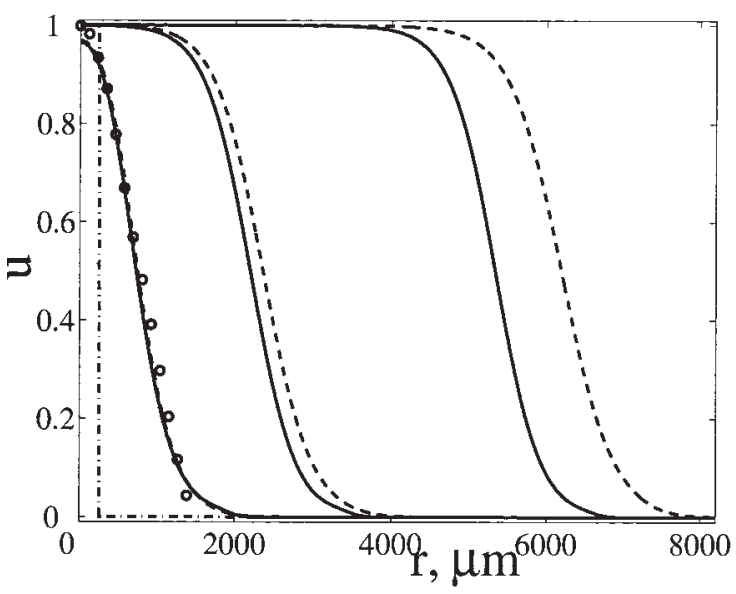

Two scenarios for tumor growth. We start from an initially sharp density profile (dash-dotted line). Time series of the cells density profiles, obtained from the numerical solution of the full system Eq. (2) are denoted by the solid lines. The profiles are obtained at times $t_{1}=5.5, t_{2}=$ 11.0, and $t_{3}=22.0$. Profiles, obtained from the diffusion-only model $\left(\chi_{1}=0\right)$ are shown by the dashed lines $\left(t_{1}=6, t_{2}=12\right.$, and $t_{3}=$ 24 , correspondingly.) Circles denote the experimental density profile. Despite the similarity between the two theoretical density profiles in the early stage (note that both of them are in a good agreement with the experimental profile), the two scenarios are distinguishable at larger times. For convenience, the distance from the center of tumor is measured in physical units $(\mu \mathrm{m})$. The parameters for the full model are: $D=$ $100, \chi_{1}=10, u_{1}=0.25, c_{1}=0.3$, and $\beta=700$. The parameters for the diffusion-only model are: $\chi_{1}=0, u_{1}=0.1$.

models do not account for growth inhibition, and further long-time in vitro experiments with brain tumor cells are needed to check the significance of this effect.

\section{SUMMARY AND DISCUSSION}

In this work, we analyzed two different mechanisms of tumor growth. The first is diffusion (random cell motion), whereas the second is chemotaxis, directed cell motion that occurs along the gradient of concentration of chemoattractant. In the experiments (Stein et al., unpublished results), the radius of the invasive region grows faster than the radius of the inner proliferation zone. Our results suggest that this regime is a transient. The model supports a propagating front, so as time increases, the expansion velocities of the two zones should tend to the same value.

We have shown that the experimental density profile can be reproduced both in the model that includes only diffusion, and a model that includes both cell diffusion and chemotaxis. However, the large time behavior is not the same, and the velocities of the propagating fronts in these two models are different. We believe our predictions would be verified if long-time experiments (2-3 weeks) could be performed beyond the transient regime. 
It is known that GBMs frequently contain amplification of mutations of the epidermal growth factor receptor (EGFR) gene. The most common mutation results in a truncated receptor tyrosine kinase known as $\triangle$ EGFR that signals constitutively and promotes GBM growth. Experiments with these mutant cells show a qualitatively different behavior (Stein et al., unpublished results) from the wild type that we have discussed here. Mutant type cells are organized in tenuous branches (Stein et al., unpublished results; [4]). This can be interpreted as an indication of an instability of a spherically symmetric propagating front. Analogous instabilities were studied in the theory of combustion [10], as well as in the context of self-organization of microorganisms $[11,12]$. One way of modeling this branching instability is assuming homotype attraction between the cells [5]. Another possibility is to assume a nonlinear diffusion, where the diffusion coefficient increases with the density of the cells, as was proposed for growth of bacterial colonies $[11,12]$. Understanding the mechanisms that lead to the instability in the context of tumor growth can be an interesting direction of the future work.

\section{Acknowledgments}

We thank the Michigan Center for Theoretical Physics for support. E.K. is grateful to Thomas Deisboeck, Baruch Meerson, and Sergey V. Shmelkov for many useful discussions. This research was supported by NIH Bioengineering Research Partnership grant R01 CA085139-01A2.

\section{REFERENCES}

1. Surawicz, T.S.; Davis, F.; Freels, S.; Laws, E.R.; Menck, H.R. Brain tumor survival: Results from the National Cancer Data Base. J Neurooncol 1998, 40, 151-160.

2. Davis, F.G.; Freels, S.; Grutsch, J.; Barlas, S.; Brem, S. Survival rates in patients with primary malignant brain tumors stratified by patient age and tumor histological type: An analysis based on Surveillance, Epidemiology, and End Results (SEER) data, 1973-1991. J Neurosurg 1998, 88, 1-10.

3. Demuth, T.; Berens, M.E. Molecular mechanisms of glioma cell migration and invasion. J Neurooncol 2004, 70, 217-228.

4. Deisboeck, T.S.; Berens, M.E.; Kansal, A.R.; Torquato, S.; StemmerRachamimov, A.0.; Chiocca, E.A. Pattern of self-organization in tumour systems: Complex growth dynamics in a novel brain tumour spheroid model. Cell Prolif 2001, 34, 115-134.

5. Sander, L.M.; Deisboeck, T.S. Growth patterns of microscopic brain tumors. Phys Rev E 2002, 66, 051901.

6. Giese, A.; Loo, M.A.; Tran, N.; Haskett, D.; Coons, S.W.; Berens, M.E. Dichotomy of astrocytoma migration and proliferation. Int $\mathrm{J}$ Cancer 1996, 67, 275-282.

7. Giese, A.; Bjerkvig, R.; Berens, M.E.; Westphal, M. Cost of migration: Invasion of malignant gliomas and implications for treatment. J Clin Oncol 2003, 21, 1624-1636.

8. Murray, J.D. Mathematical Biology; Springer-Verlag: Berlin, 1989.

9. Freyer, J.P. Role of necrosis in regulating the growth saturation of multicellular spheroids. Cancer Res 1988, 48, 2432-2439.

10. Sivashinsky, G.I. Instabilities, pattern-formation, and turbulence in flames. Annu Rev Fluid Mech 1983, 15, 179-199.

11. Ben-Jacob, E.; Cohen, I.; Levine, H. Cooperative self-organization of microorganisms. Adv Phys 2000, 49, 395-554.

12. Golding, I.; Kozlovsky, Y.; Cohen, I.; Ben-Jacob, E. Studies of bacterial branching growth using reaction-diffusion models for colonial development. Physica A 1998, 260, 510-554. 